$562 \cdot 31$

\title{
puente aligerado sobre el río Storms
}

\author{
RICCARDO MORANDI, ingeniero
}

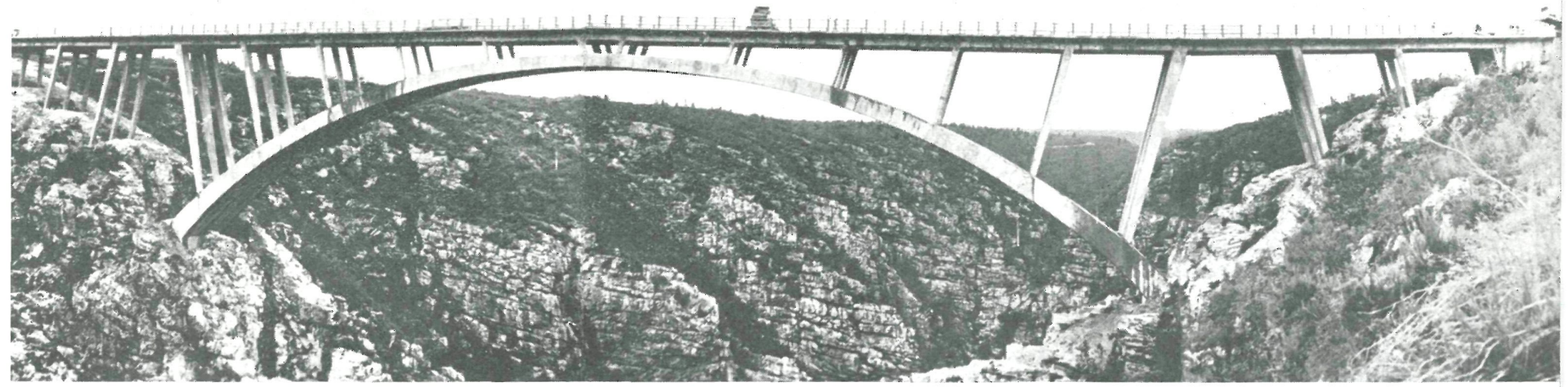

S I $\mathbf{N}$ O

Descripción del puente aligerado, de hormigón armado, construído sobre el río Storms Africa del Sur), en arco, de $100,58 \mathrm{~m}$ de luz y $20,11 \mathrm{~m}$ de flecha, que presenta la particularidad de soportar el tablero por medio de soportes oblicuos $y$ haberse empleado un procedimiento constructivo notable, que se podría incluir en los sistemas des ejecución por medio de autocimbras.

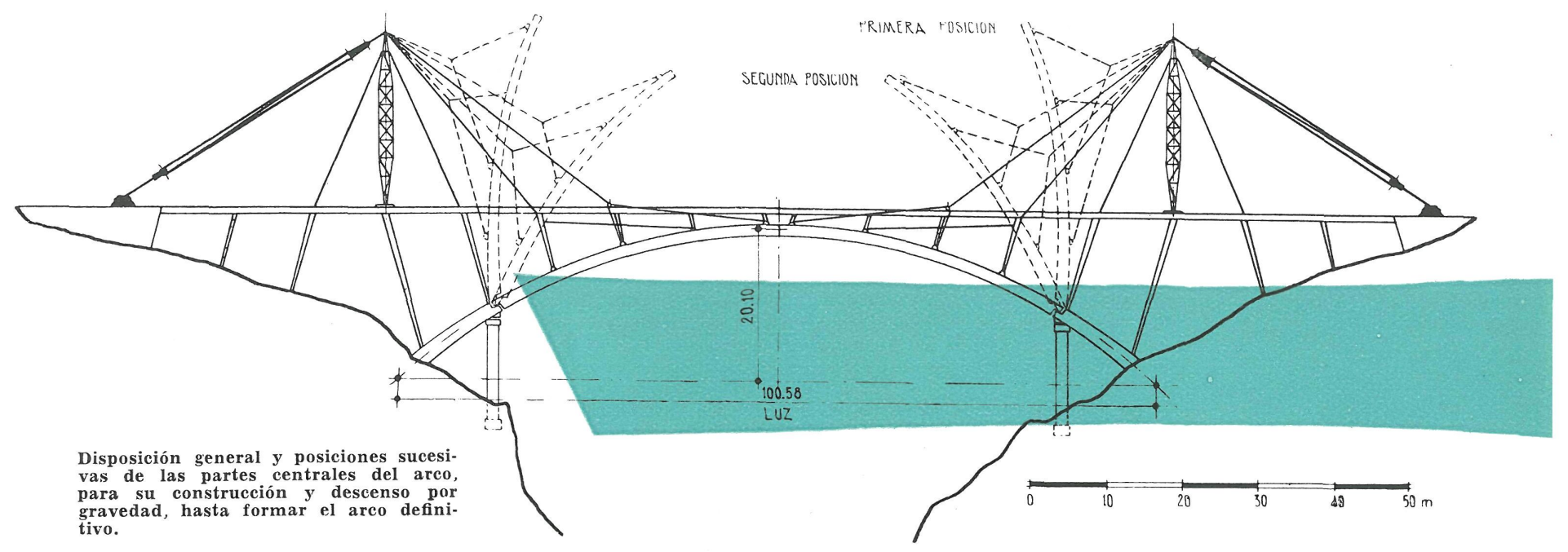

I NSTITUTO TECNICO DE LA CONSTRUCCION Y DEL CEMENTO 


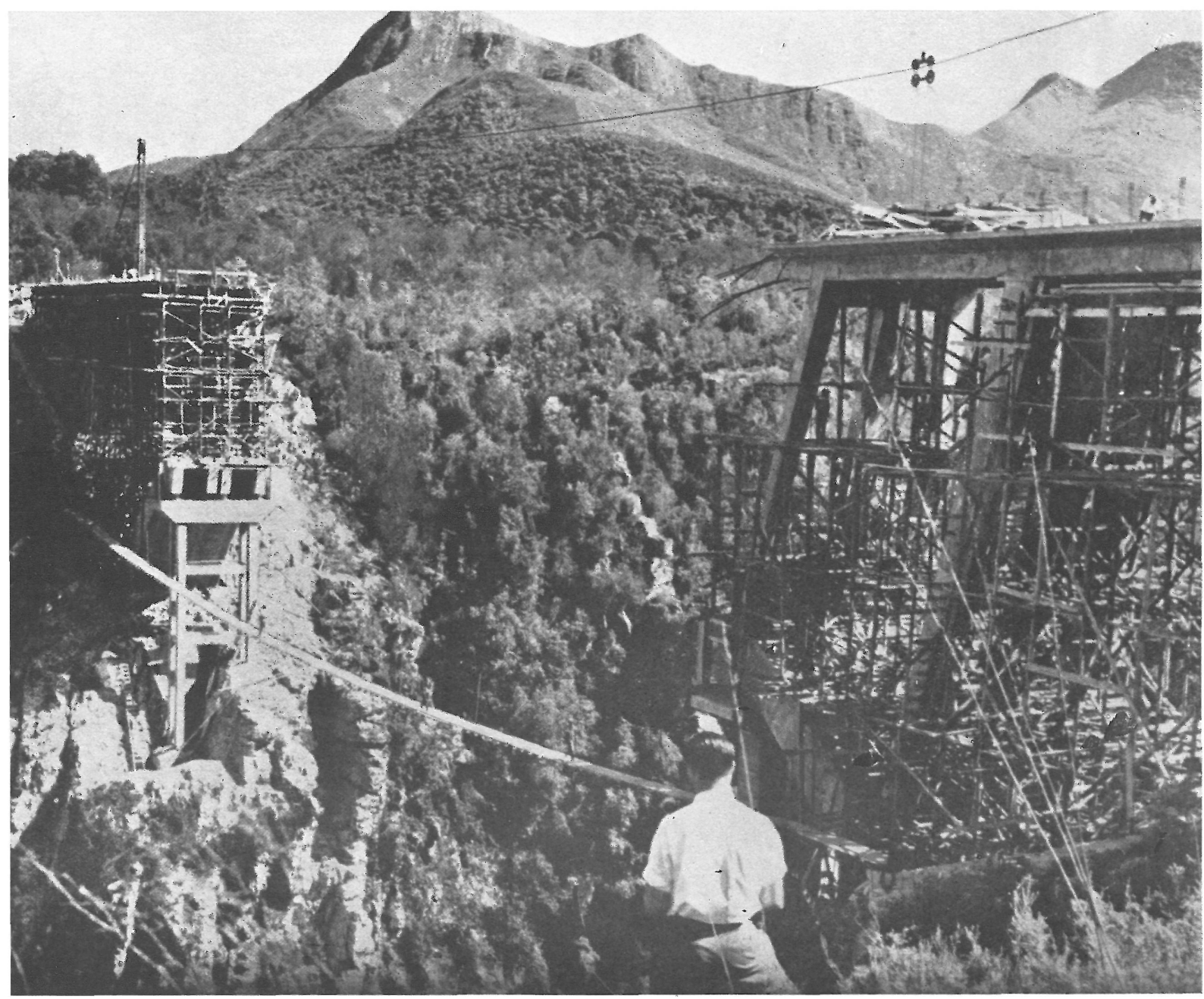

Un momento de la tramos laterales del tramos laterales del pasarela de servicio.

\section{Generalidades}

La carretera que une Port Elisabeth con Cape Town (Africa del Sur), cruza el río Storms en una garganta muy acantilada que alargaba considerablemente el trazado de dicho camino. Con objeto de mejorar el trazado, y teniendo en cuenta la dificultad local para encimbrar por los métodos ordinarios, así como la exclusión de una estructura metálica, el autor de este trabajo, honrado con la redacción del proyecto de esta obra, presentó un proyecto de puente de hormigón armado, con un arco de 100,58 m de luz, 20,11 m de flecha, de sección hueca, aligerado, soportando el tablero por medio de soportes oblicuos, apoyados unos sobre e! arco y otros sobre el terreno.

\section{Cálculo y estabilidad de la obra}

Las sobrecargas previstas para el puente se ajustan a la norma número 577 del Ministerio de Transportes inglés. El módulo de elasticidad del hormigón admitido es de $300.000 \mathrm{~kg} / \mathrm{cm}^{2}$; de -4 a $43^{\circ} \mathrm{C}$, las variaciones extremas de temperatura, y una carga de $147 \mathrm{~kg} / \mathrm{m}^{2}$ debido a la acción del viento. La línea general estética de la obra se cuidó convenientemente para niejorar su aspecto general.

El arco se calculó siguiendo el método de la elipse central de elasticidad; la curva acioptada es muy parecida a la resultante de un funicular trazado considerando el peso propio y sobrecargas. Las cargas de trabajo de los materiales en la clave son de $30,70 \mathrm{~kg} / \mathrm{cm}^{2}$ para el hormigón y $1356 \mathrm{~kg} / \mathrm{cm}^{2}$ para las armaduras.

Los montantes de los pórticos que constituyen los soportes inclinados del tablero, se han articulado en su base para no inducir momentos secundarios en el arco. La solución de nicntantes oblicuos presenta una doble ventaja, ya que disminuye el empuje y se presta favorablemente a resistir contra la acción del viento. 


\section{Descripción de la obra}

El arco se subdividió en cuatro partes para su construcción: dos de éstas corresponden a los arranques y las otras dos-cnnstruídas en posición casi vertical y apoyadas sobre soportes provisionales en correspondencia con las dos articulaciones formadas en las extremidades de los arranques del arco-se las descendió por giro alrededor de las articulaciones, sirviéndose de un aparejo especial, hasta hacer coincidir sus extremidades libres en la clave.

Estas dos partes centrales, de unos $45 \mathrm{~m}$ de longitud cada una, especie de autocimbra, se han subdividido, a su vez, en otras dos partes, formando así dos segmentos de arco, cada uno de los cuales, articulado en su pie para el giro que les ha de llevar a la posición definitiva, está formado por dos almas o nervios de hormigón armado, que se han arriostrado entre sí por medio de codales de intradós y extradós que le dan un aspecto de cajón en arco. El intradós de estas partes va provisto de un encofrado, que permitirá hormigonar la rosca de intradós.

Una vez en posición, después del giro, de las dos partes centrales, la estructura, aún no terminada, actúa ya como arco. A continuación se procede a transformar la articulación en un elemento rígido: a hormigonar la rosca de intradós fondo la rosca del extradós. Como se comprenderá fácilmente, la sección del arco así formado la rosca del extradós. Como se comprenderá fácilmente, la sección del arco así formado central de la sección está formada por dos tabiques adosados, cada uno de los cuales corresponde al alma interior de los dos cajones en arco.

Sobre el extradós y terreno se apoyan los soportes oblicuos que sostienen el tablero, formando pórticos hiperestáticos de tres montantes de forma troncopiramidal. En los cabezales de estos pórticos descansan tres vigas longitudinales, de sección variable, decreciente de estribos a la clave. Sobre estas vigas se ha hormigonado el tablero, de $8,18 \mathrm{~m}$ de anchura total, que vuela a los dos lados.

La sección del arco tiene una anchura variable, de $7,10 \mathrm{~m}$ en arranques a $6,30 \mathrm{~m}$ en la clave; el espesor de las dos almas laterales es también variable, de 0,61 $\mathrm{m}$ de arranques a $0,28 \mathrm{~m}$ en la clave $\mathrm{y}$, por tanto, el alma central varía de 1,22 a $0,56 \mathrm{~m}$. Las dos roscas de intradós y extradós están constituídas por losas, cuyo espesor varía de 0,42 m en arranques a $0,29 \mathrm{~m}$ en la clave.

La altura o canto del arco es de $2,55 \mathrm{~m}$ en imposta, y va disminuyendo regularmente hasta $1,21 \mathrm{~m}$ en la clave.

Las vigas longitudinales tienen $0,98 \mathrm{~m}$ de canto y una anchura variable de $0,73 \mathrm{~m}$ en el estribo y $0,30 \mathrm{~m}$ en la parte central del tablero, cuyo espesor es de $17,8 \mathrm{~m}$.

\section{Fases sucesivas de la construcción de la obra}

La ejecución de la obra se ha subdividido en varias fases: Se empezó construyendo los arranques y soportes provisionales que los debian sostener, servir de apoyo para la construcción y giro de las partes centrales del arco y, finalmente, ser demolidos. La altura de estos soportes fué, respectivamente, de 23,4 y $18,20 \mathrm{~m}$.

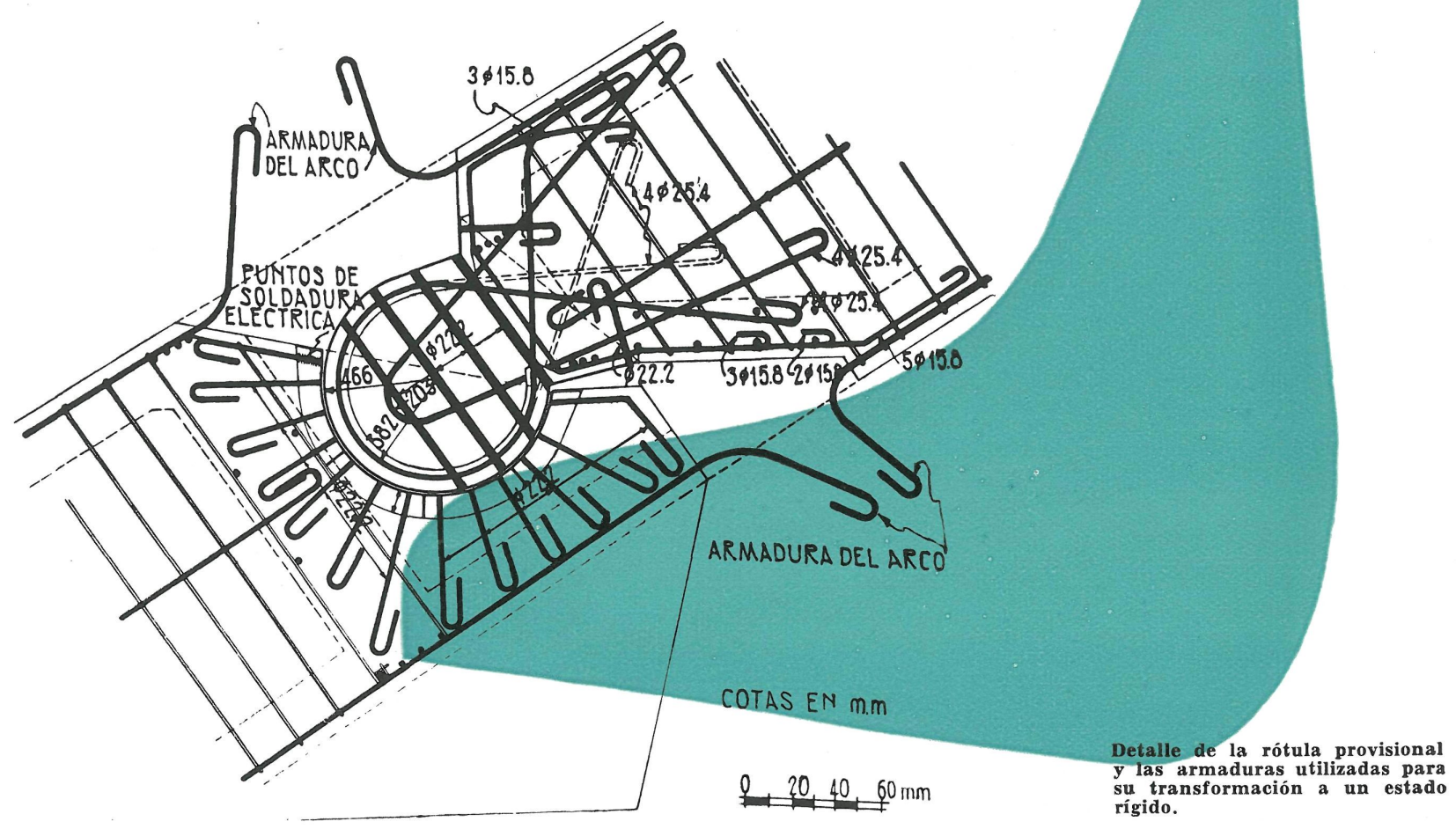




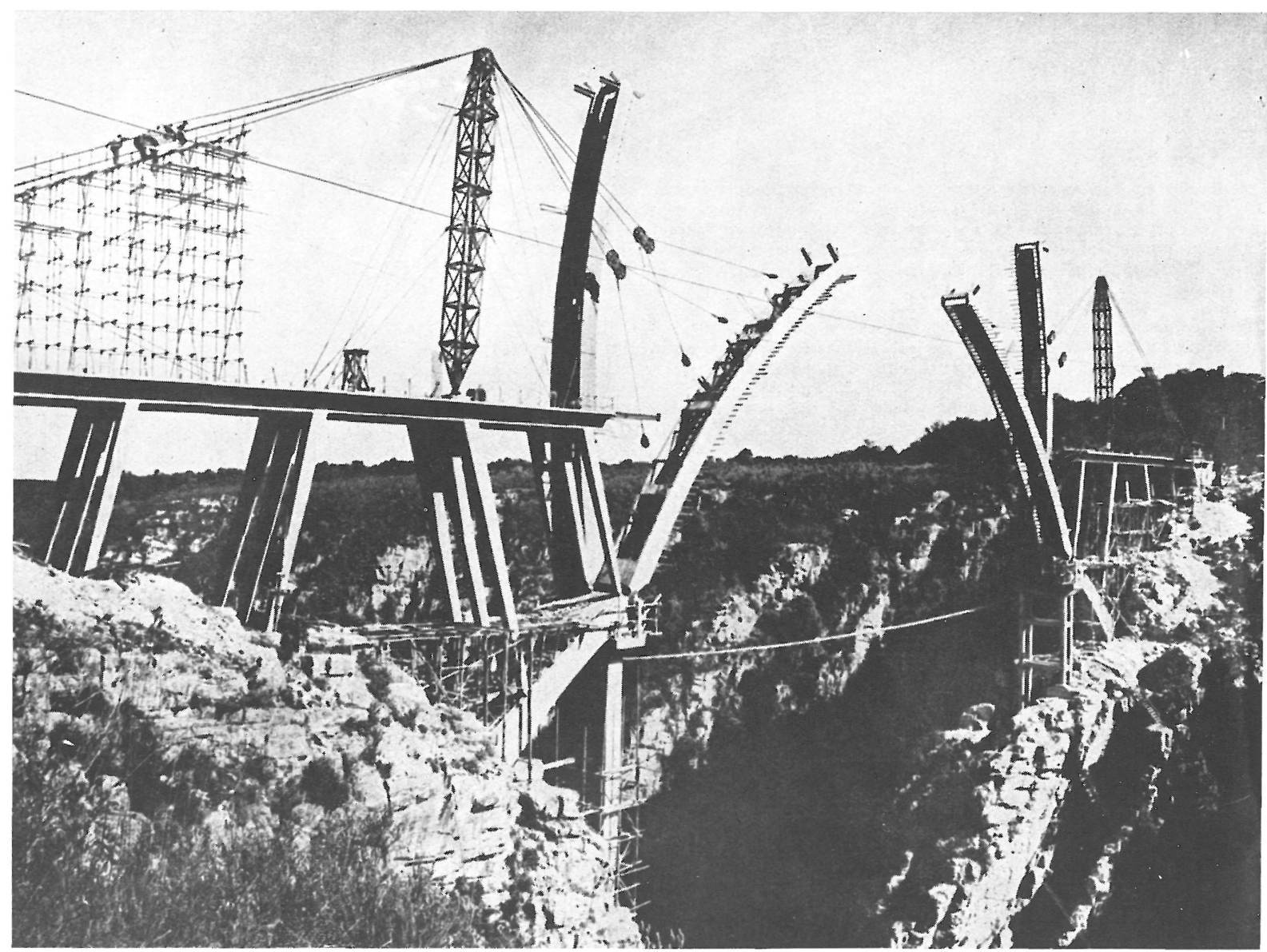

Los dos elementos o semiarcos que constituyen Ia hallan hallan en el curvolteo.

Diagrama de momentos de un momento de semiarco, debidos al peso propio y al efecto de compresión de los cables tirantes provisionales.

Terminada esta parte de la obra, se procedió a la construcción, a uno y otro lado del puente, de los estribos y tramos hasta los arranques.

La fase siguiente consistió en construir las dos partes centrales del arco, subdivididas, a su vez, en otras dos partes longitudinales, independientes, de unas 80 toneladas de peso cada una, en posición casi vertical. Los rodillos de la articulación provisional están formados por tubos concéntricos, de $0,91 \mathrm{~m}$ de diámetro, cuya disposición y unión con el resto de las armaduras del arco presentó ciertas dificultades.

La cuarta fase constructiva consistió en la rotación de estas partes centrales del arco hasta lograr su unión en la clave. La operación se llevó a cabo mediante tornos hidráulicos y un aparejo provisto de una pluma que se instaló sobre la parte de tablero ya construída.

El descenso de estas partes de arco se realizó por gravedad y freno de retención, siguiendo un control minucioso para evitar, en la medida de lo posible, toda clase de deformaciones. De la posición inicial a la segunda intermedia no tenían gran importancia los efectos de flexión por peso propio; pero, de esta última posición a la definitiva estos efectos se hacían sentir excesivamente. Para evitar este inconveniente se colocaron unos cables entre las extremidades de los segmentos de arco, que, convenientemente tesados, lograron reducir los efectos nocivos de flexión antes enunciados.

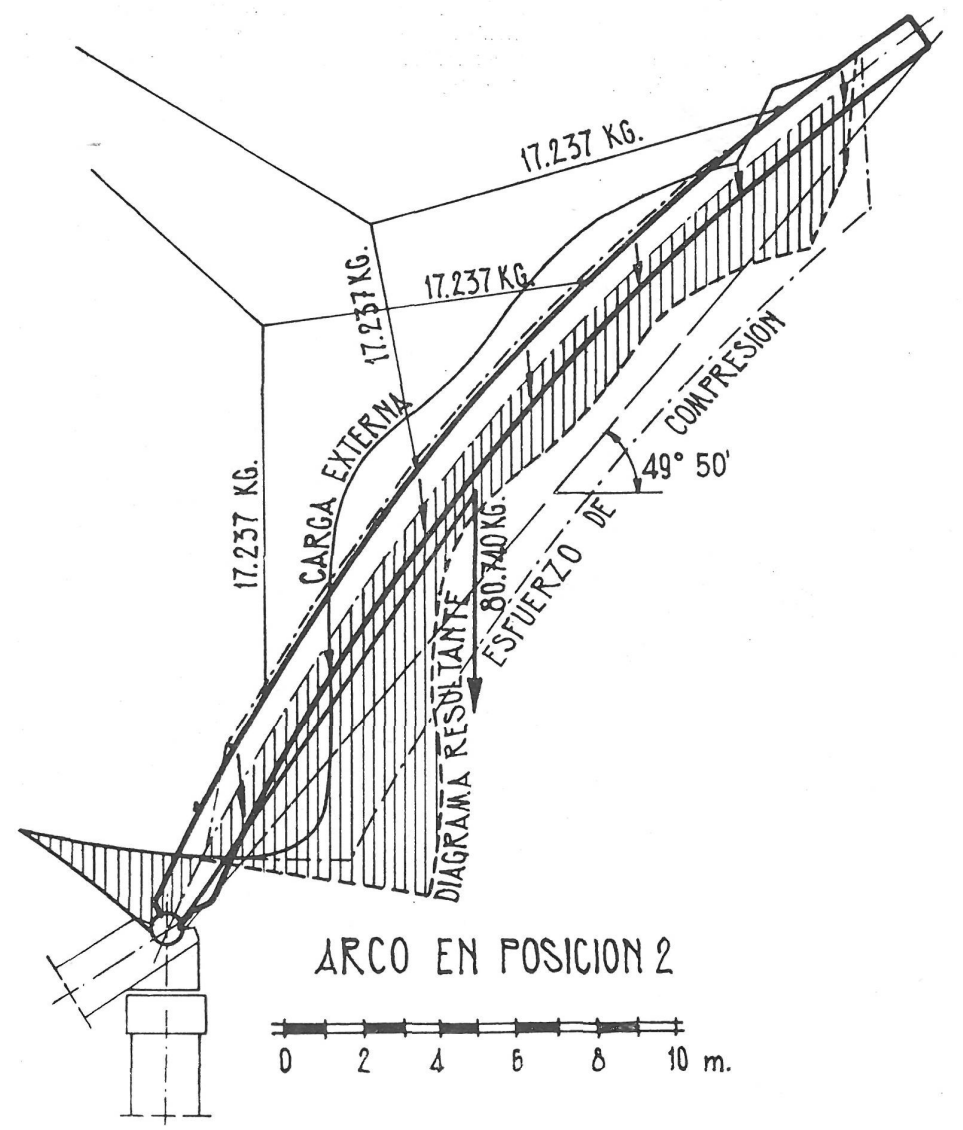




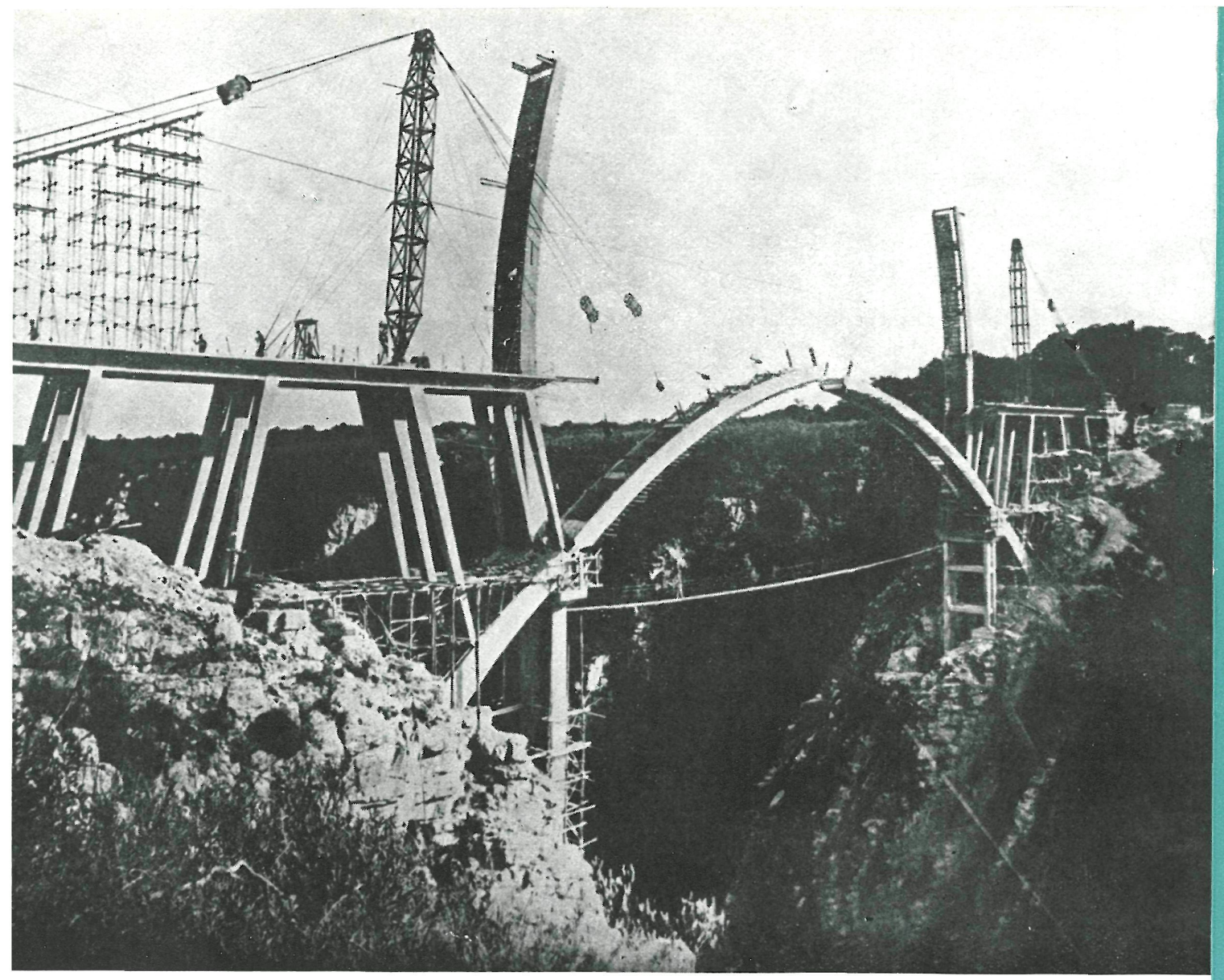

Dos de las partes centrales autocimbras del arco, en un momento de su rotación para llegar a la po. sición definitiva.

Diagrama de momen. tos de un elemento de semiareo, en la debidos al pese propio $y$ al peso procompresión de los tiantes provisionales.

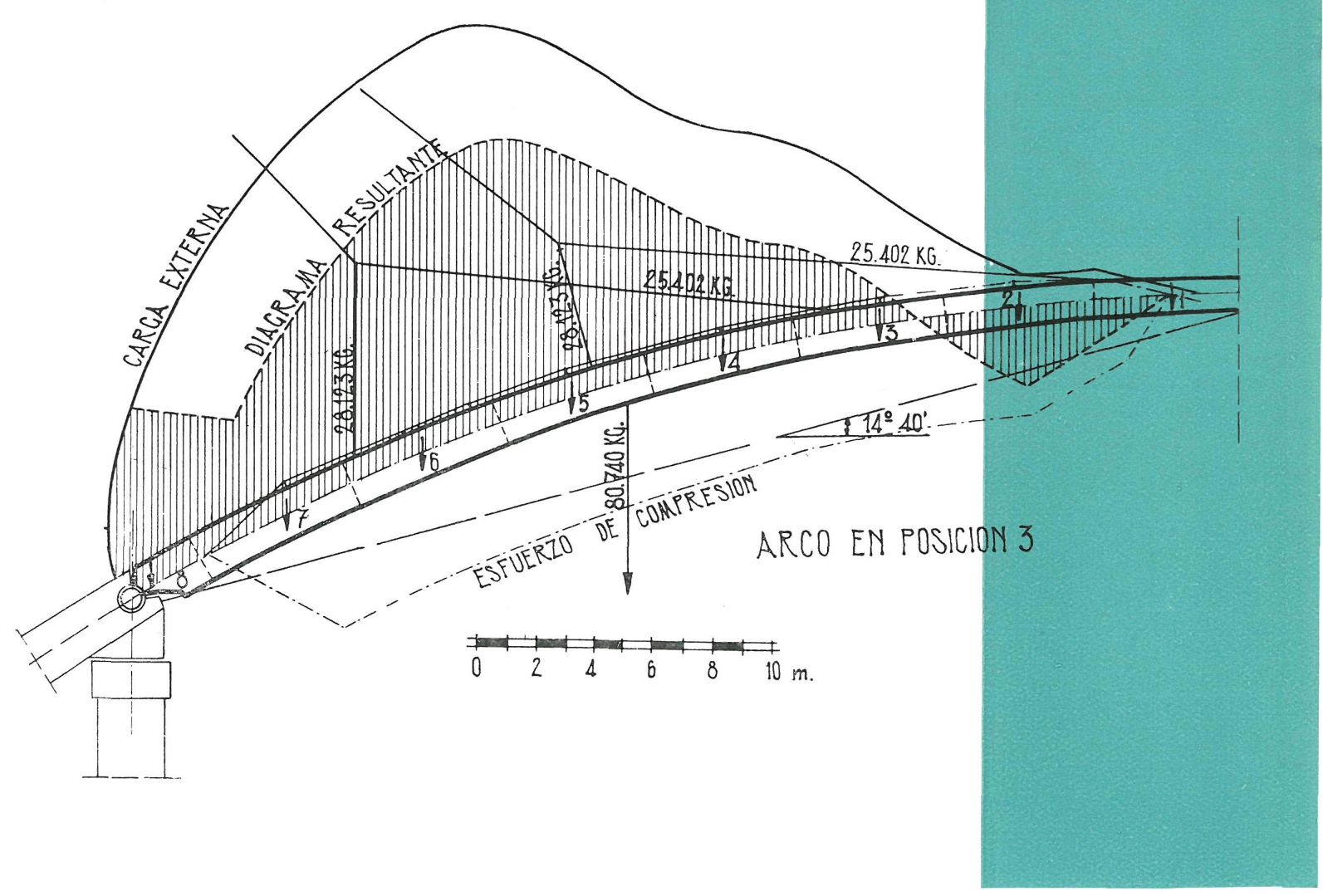




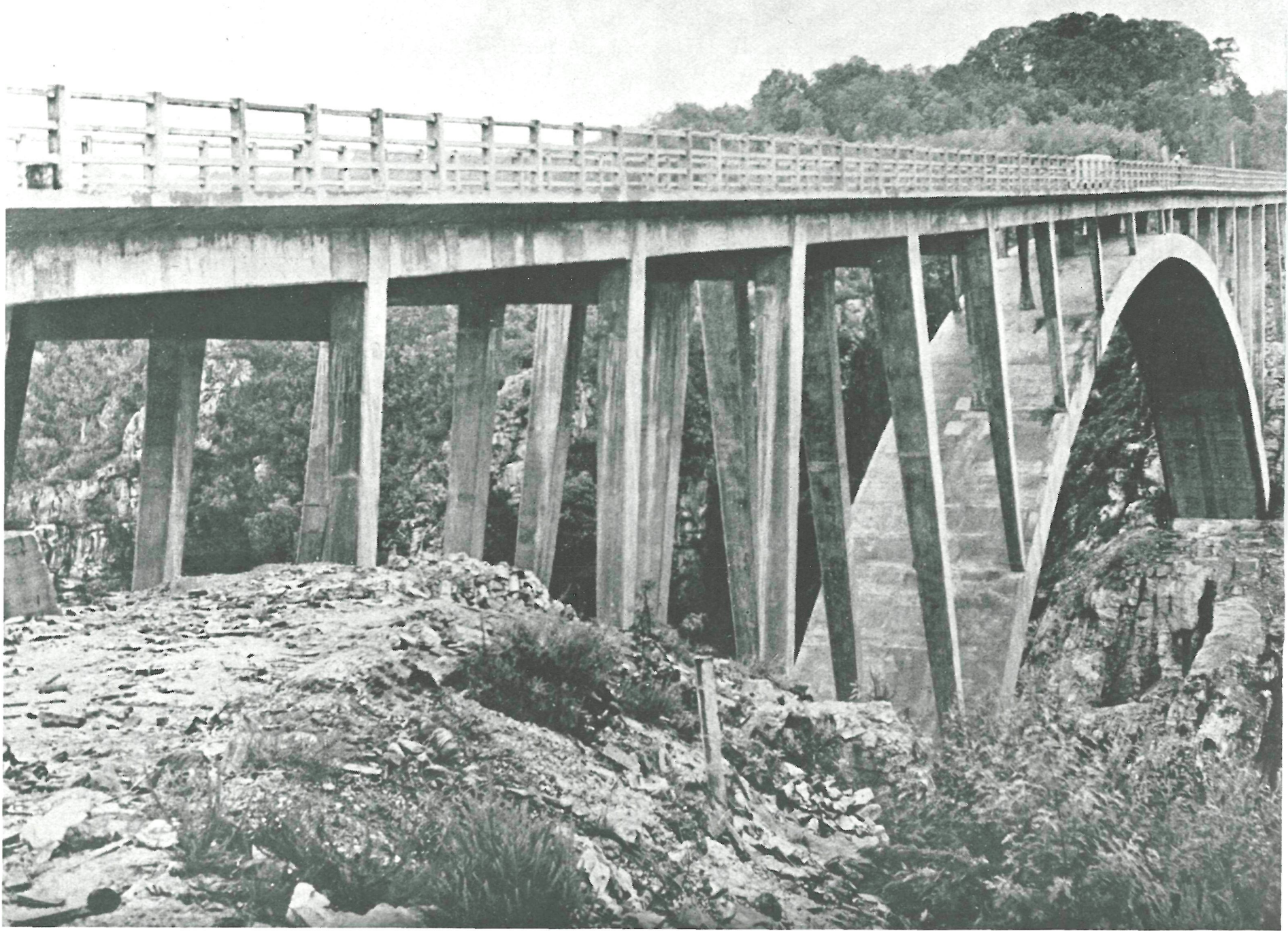

Aspecto general del puente ya terminado.
La unión en la clave se realizó, provisionalmente, con unos perfiles, de $1 \mathrm{~m}$ de longitud, que se hallaban anclados en las extremidades de estos segmentos de arco, dando lugar al final, después de aflojar los cables, a un arco de tres articulaciones, momentáneamente. Las operaciones de descenso, de una a otra de las posiciones sucesivas hasta llegar a la final, no presentaron dificultades.

La operación siguiente, o quinta fase constructiva, consistió en suprimir el efecto proLa operación siguiente, o quinta fase constructiva, consistio en suprimir el efecto prode intradós y extradós, dando al arco su forma celular, y, finalmente, destruir los apoyos provisionales de los arranques. Esta última operación no produjo descenso importante en la clave.

La sexta y última fase constructiva consistió en terminar los tramos de imposta y clave que soportan al tablero, así como la terminación de éste y los antepechos.

Esta obra fué ejecutada siguiendo los principios propuestos y sin tener que lamentar contrariedad alguna respecto a accidentes o desperfectos materiales. 